\title{
Influência do envelhecimento termo-oxidativo nas propriedades mecânicas e de amarelamento de blendas de poliestireno com borracha reciclada de estireno-butadieno (SBR)
}

\author{
Influence of thermo-oxidative aging \\ on the mechanical and yellowing properties \\ of polystyrene blends with recycled \\ styrene-butadiene rubber (SBR)
}

Carlos Bruno Barreto Luna ${ }^{1}$, Felipe Belchior Calheiro Gomes ${ }^{1}$, Danilo Diniz Siqueira ${ }^{1}$, Edcleide Maria Araújo ${ }^{1}$

\footnotetext{
${ }^{1}$ Unidade Acadêmica de Engenharia de Materiais, Universidade Federal de Campina Grande, 58429-140, Campina Grande , Paraíba, Brasil. e-mail: brunobarretodemaufcg@hotmail.com, edcleide.araujo@ufcg.edu.br
}

\section{RESUMO}

Este trabalho teve como objetivo avaliar o envelhecimento termo-oxidativo, nas propriedades mecânicas e de índice de amarelamento (YI), de blendas de poliestireno com borracha reciclada (SBRr), compatibilizadas. As blendas foram preparadas, inicialmente, em uma extrusora de rosca dupla corrotacional e, posteriormente, os grânulos extrudados foram moldados por injeção. Para efeito comparativo, o poliestireno de alto impacto comercial (HIPS) foi processado nas mesmas condições das blendas. Os resultados da caracterização do SBRr indicaram, em sua composição, cargas de sílica, carbonato de cálcio, talco e óxido de zinco, enquanto que, por termogravimetria, verificou-se duas etapas de decomposição térmica. Verificou-se que a resistência ao impacto diminuiu, significativamente, nos 15 dias iniciais do envelhecimento, e, subsequentemente, apresentou uma tendência de estabilização com pequenas oscilações. As propriedades de módulo de elasticidade, resistência e alongamento na ruptura sob tração apresentaram o mesmo comportamento da resistência ao impacto, redução acentuada nos 15 dias inicias, seguida de estabilização, com o decorrer do envelhecimento. Quando a blenda foi compatibilizada com 5\% de estireno-butadieno-estireno (SBS), foram observadas as melhores propriedades, antes e após o envelhecimento, apresentando resistência ao impacto equiparáveis ao HIPS. A perda de massa das blendas indicou extração de componentes voláteis. Por índice de amarelamento (YI), observou-se, nas blendas, um aumento brusco nos 15 dias iniciais e, em seguida, estabilidade, com uma taxa de amarelamento praticamente constante. Até 30 dias de envelhecimento, o HIPS apresentou uma baixa taxa de amarelamento, e, posteriormente, ocorreu uma intensificação no YI. No geral, as blendas obtidas por mistura mecânica apresentaram comportamento similar ao HIPS. Porém, somente quando a blenda foi compatibilizada com SBS que o comportamento de impacto apresentou o mesmo patamar do HIPS.

Palavras-chave: envelhecimento, termo-oxidação, blendas poliméricas, propriedades mecânicas, índice de amarelamento.

\section{ABSTRACT}

The objective of this study was to evaluate thermo-oxidative aging in the mechanical properties and yellowing index (YI) of polystyrene blends with compatibilized recycled rubber (SBRr). The blends were initially prepared in a co-rotating double screw extruder, after which the extruded granules were injection molded; high-impact polystyrene (HIPS) was processed under the same conditions for comparison. The SBRr characterization results revealed composition fillers of silica, calcium carbonate, talc, and zinc oxide, while thermogravimetry demonstrated two thermal decomposition stages. It was found that impact strength decreased significantly during the initial 15 days of aging and then showed a tendency to stabilize with small oscillations. Properties related to the modulus of elasticity, strength, and elongation at tensile rupture were similar to those of impact strength: a marked reduction in the initial 15 days followed by stabilization with aging. When a blend was compatibilized with 5\% styrene-butadiene-styrene (SBS), optimal properties were observed before 
and after aging, indicating impact resistance comparable to HIPS. The blends' loss of mass suggested volatile component extraction. In terms of the YI, the blends showed a sudden increase in the first 15 days of aging before stabilizing at a practically constant yellowing rate. Up to 30 days of aging, HIPS presented a low yellowing rate, after which point the YI intensified. In general, the blends obtained via mechanical mixing exhibited behavior similar to HIPS; for the blend compatibilized with SBS, however, the impact behavior was identical to that of HIPS.

Keywords: aging, thermo-oxidation, polymer blends, mechanical properties, yellowing index.

\section{INTRODUÇÃO}

Degradação é o conjunto de reações químicas que alteram a qualidade de interesse de um material polimérico, envolvendo quebra de ligações primárias da cadeia principal do polímero e formação de outras, com consequente mudança da estrutura química e redução da massa molar [1,2].

Os materiais poliméricos podem sofrer degradação sob ação de temperatura, luz, umidade e agentes químicos. Quando um polímero é mantido numa dada temperatura, mesmo que moderada, ou em ciclos térmicos que varie sistematicamente, e repetidamente por períodos de tempos longos, ele pode ser degradado. Além disso, quando o polímero se encontra em uma atmosfera oxidante, sua degradação é mais rápida, sendo denominada termo-oxidação ou envelhecimento termo-oxidativo [3]. Geralmente, a degradação causa um efeito deletério nas propriedades mecânicas dos materiais poliméricos, e altera a sua cor. Para isso, dependendo da estrutura molecular, um polímero pode tornar-se vulnerável aos efeitos do ambiente, como o calor, o oxigênio, a umidade, as emissões de poluentes atmosféricos e a radiação [4]. Os polímeros insaturados, como as borrachas, são facilmente oxidáveis, tendo como elo fraco as ligações duplas [5]. Os copolímeros e as blendas tenacificadas com borrachas têm grande importância comercial, devido à sua alta resistência ao impacto, entre outras propriedades [6-9]. Portanto, pesquisas no sentido de estudar a influência do envelhecimento termo-oxidativo de copolímeros ou blendas reforçadas com borrachas, são convenientes para entender a durabilidade desses materiais, bem como, as influências nas propriedades.

VILAPLANA et al. [10] estudaram a degradação termo-oxidativa do poliestireno de alto impacto (HIPS ou PSAI), sendo observada uma queda de $84 \%$ na elongação na ruptura, após quatro dias de envelhecimento acelerado a $90{ }^{\circ} \mathrm{C}$. Nos estudos de SARON e FELISBERTI [11] sobre envelhecimento térmico, submetido a $75^{\circ} \mathrm{C}$ em estufa ventilada, a formação de carbonilas é quase imperceptível, no HIPS. Os radicais livres que são formados, tanto na superfície como no interior da amostra, provocaram reações de reticulações e uma pequena oxidação superficial. Na literatura [12,13], a fase polibutadieno (PB), que contem ligações duplas, é reportada como sendo o sítio inicial de processos foto-oxidativos, termo-oxidativos e temomecânicos de degradação de copolímeros estirênicos, uma vez que a presença da ligação dupla reduzirá a energia da ligação $\mathrm{C}-\mathrm{H}$ adjacente.

O mecanismo de envelhecimento por termo-oxidação do poliestireno de alto impacto (HIPS) já é bem estabelecido na literatura [10,11,14]. Entretanto, não foram localizados, na literatura de polímeros, estudos sobre termo-oxidação de blendas de poliestireno (PS) utilizando borracha reciclada de SBR, obtidas por meio de misturas mecânicas. Na literatura [15-18], é reportado que essas borrachas vulcanizadas são constituídas de uma mistura complexa de SBR (copolímero de butadieno-estireno), cargas, aditivos de processamento, agentes de cura e estabilizantes. Sendo assim, é conveniente estudar esse composto reciclado de SBR e, consequentemente, analisar o real potencial tecnológico frente à estabilidade termo-oxidativa.

A degradação da borracha está relacionada com a cisão molecular, que resulta em cadeias menores e num maior número de terminais de cadeia, e/ou em reticulação, o que gera uma estrutura em rede fortemente ligada. É importante considerar que, com a cisão das cadeias, a viscosidade decresce, mas no caso da reticulação, o material torna-se mais rígido [19]. Nos estudos de LUCAS et al. [20], foram propostas duas reações básicas, que levaram às modificações da estrutura química das borrachas conforme Figura 1. 


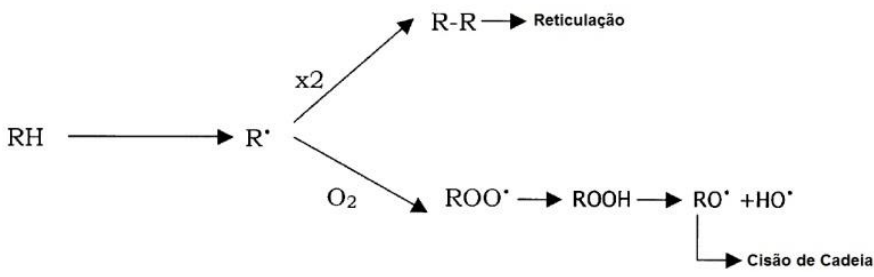

Figura 1: Reações básicas que levam às modificações da estrutura química de elastômeros [20].

A degradação de blendas poliméricas é uma área de grandes dificuldades, por tratar-se de uma mistura de dois componentes distintos, com possibilidade de interações específicas. Geralmente, quando se desenvolve uma blenda com uma matriz, fase dispersa e compatibilizante com grupos químicos similares, aumenta-se a complexidade do entendimento dos efeitos degradativos. O efeito dos agentes compatibilizantes no comportamento de blendas, relativo à degradação termo-oxidativa, é muito pouco reportado na literatura [2]

Neste trabalho, verificou-se o efeito do envelhecimento termo-oxidativo em blendas de poliestireno, reforçadas com um composto de borracha reciclada da indústria de calçados. Essas foram compatibilizadas com estireno-butadieno-estireno (SBS) e estireno-etileno/butileno-estireno (SEBS), e as propriedades mecânicas e de amarelamento foram avaliadas e comparadas com o HIPS comecial.

\section{MATERIAIS E MÉTODOS}

\subsection{Materiais}

As blendas foram preparadas a partir do poliestireno cristal (PS), comercializado com o código U288 ${ }^{\circledR}$ e índice de fluidez de $3 \mathrm{~g} / 10 \mathrm{~min}\left(200^{\circ} \mathrm{C} / 5 \mathrm{~kg}\right)$, fornecido na forma de grânulos e fabricado pela Unigel S.A (Brasil). Foi utilizado como carga um composto reciclado de borracha vulcanizada de estireno-butadieno (denominado de SBRr), na forma de pó, de cor branca, proveniente da indústria de calçados São Paulo Alpargatas S.A., sediada na cidade de Campina Grande-PB. Foram utilizados como agentes compatibilizantes o copolímero tribloco linear estireno-butadieno-estireno (SBS), na forma de grânulos, comercializado com o código D1101B ${ }^{\circledR}$, contendo $31 \%$ de estireno e índice de fluidez (IF) menor que $1 \mathrm{~g} / 10 \mathrm{~min}$, fornecido pela Activas S.A (Brasil); e o copolímero estireno-(etileno-butileno)-estireno (SEBS), na forma de pó, sob o código G1652 ${ }^{\circledR}$, contendo $30 \%$ de estireno e índice de fluidez (IF) de $5 \mathrm{~g} / 10 \mathrm{~min}$, fornecido pela Kraton.

Para fins de comparação com as blendas, foi utilizado o poliestireno de alto impacto (HIPS ou PSAI), comercializado com o código Styron $478^{\circledR}$, índice de fluidez de $6 \mathrm{~g} / 10 \mathrm{~min}\left(200^{\circ} \mathrm{C} / 5 \mathrm{~kg}\right)$, fornecido na forma de grânulos, e fabricado pela Dow Plásticos.

\subsection{Preparação das Blendas}

Antes da preparação das blendas, o composto de borracha reciclado, advindo da indústria, foi peneirado em malha 18 mesh $(1 \mathrm{~mm})$, sendo utilizado, nessa malha, o pó passante. A Tabela 1 ilustra as proporções em massa $(\%)$ das composições que foram utilizadas no desenvolvimento das blendas ternárias, binárias e do HIPS.

Tabela 1: Composições das blendas ternárias, binárias e do HIPS.

\begin{tabular}{lcccc}
\hline \multicolumn{1}{c}{ Materiais } & $\begin{array}{c}\text { PS } \\
(\% \text { em massa })\end{array}$ & $\begin{array}{c}\text { SBRr } \\
(\% \text { em massa })\end{array}$ & $\begin{array}{c}\text { SBS ou SEBS } \\
(\% \text { em massa })\end{array}$ & $\begin{array}{c}\text { HIPS } \\
\text { (\% em massa })\end{array}$ \\
\hline HIPS & - & - & - & 100 \\
PS/SBRr & 50 & 50 & - & - \\
PS/SBRr/SEBS & 47,5 & 47,5 & 5 & - \\
$(\mathbf{5 \% )}$ & 47,5 & 47,5 & 5 & - \\
PS/SBRr/SBS (5\%) & & & 5 \\
\hline
\end{tabular}

* Todos os componentes foram misturados, simultaneamente, em uma única etapa de extrusão.

As blendas binárias e ternárias foram misturadas a seco e, posteriormente, processadas em uma extrusora de rosca dupla corrotacional modular, modelo ZSK ( $D=18 \mathrm{~mm}$ e $\mathrm{L} / \mathrm{D}=40)$, da Coperion Werner \& 
Pfleiderer, com temperatura de $190^{\circ} \mathrm{C}$ nas zonas 1 e 2 , e $200^{\circ} \mathrm{C}$ nas demais zonas, velocidade de rotação da rosca de $250 \mathrm{rpm}$ e taxa de alimentação controlada de $4 \mathrm{~kg} / \mathrm{h}$, com perfil de rosca configurado com elementos de misturas distributivos e dispersivos. Após o processamento das blendas por extrusão, o material foi granulado e seco em uma estufa sem vácuo por $24 \mathrm{~h}$, em temperatura de $60^{\circ} \mathrm{C}$, e, posteriormente, com vácuo na mesma temperatura por $24 \mathrm{~h}$. O poliestireno de alto impacto (HIPS) foi processado nas mesmas condições das blendas.

O HIPS e as blendas obtidas por extrusão foram moldados por injeção em uma injetora Arburg, Modelo Allrounder 207C Golden Edition, para obtenção de corpos de prova. A temperatura de moldagem foi de $200^{\circ} \mathrm{C}$ em todas as zonas, 20 segundos de tempo de injeção e temperatura do molde de $20^{\circ} \mathrm{C}$.

\subsection{Envelhecimento na Estufa}

O envelhecimento termo-oxidativo foi conduzido em uma estufa de ar circulante a $70^{\circ} \mathrm{C}$, seguindo as recomendações da norma ASTM D5510. O tempo de envelhecimento foi definido em 60 dias, sendo dividido em quatro ciclos: $15,30,45$ e 60 dias.

\subsection{Caracterização dos Materiais}

Pó da Borracha Reciclada (SBRr)

A presença de cargas no SBRr foi determinada pela análise semiquantitativa de EDS (espectroscopia de energia dispersiva), associado a um microscópio eletrônico de varredura MEV, equipamento VEGAN 3 TESCAN, com uma voltagem de $30 \mathrm{kV}$ e sob alto vácuo. Para isso, o pó de SBRr foi calcinado em um forno elétrico Quimis, modelo Q-318M24, durante $1 \mathrm{~h}$ até $600^{\circ} \mathrm{C}$.

A termogravimetria (TG) da borracha reciclada foi obtida em um equipamento da TA Instruments SDT-Q600 Simultaneous TGA/DSC/DTA, empregando cerca de $5 \mathrm{mg}$ de amostra, razão de aquecimento de $10^{\circ} \mathrm{C} / \mathrm{min}$ e vazão do gás de $100 \mathrm{~mL} / \mathrm{min}$, partindo da temperatura de 30 até $600^{\circ} \mathrm{C}$, sob atmosfera de nitrogênio.

Blendas Ternárias, Binárias e o HIPS

O ensaio de resistência ao impacto Izod foi realizado em corpos de prova entalhados, segundo a norma ASTM D256, em um aparelho da marca Ceast modelo Resil 5,5 J, operando com martelo de 2,75 J, em temperatura ambiente $\left(\sim 23^{\circ} \mathrm{C}\right)$. Os resultados foram analisados com uma média de 5 corpos de prova.

Os ensaios de tração foram realizados em corpos de prova injetados, segundo a norma ASTM D638, utilizando uma máquina de ensaios universal da marca EMIC DL 2000, com velocidade de carregamento de $5 \mathrm{~mm} / \mathrm{min}$ e célula de carga de $2 \mathrm{kN}$. Os testes foram conduzidos à temperatura ambiente $\left(\sim 23^{\circ} \mathrm{C}\right)$, e os resultados analisados a partir da média de 5 corpos de prova.

A variação de massa das amostras foi avaliada por meio da medição da massa inicial $\left(\mathrm{M}_{0}\right)$, para 0 dias de exposição e da massa final $\left(\mathrm{M}_{\mathrm{F}}\right)$, com 60 dias de envelhecimento termo-oxidativo. As mudanças de variação de massa foram calculadas conforme a equação:

$$
\% \text { Variação de massa }=\frac{M_{F}-M_{0}}{M_{0}} * 100
$$

O índice de amarelamento (YI) do HIPS e das blendas, antes e depois da degradação termo-oxidativa, foi obtido por meio de um analisador de cor portátil FRU ${ }^{\circledR}-10 Q C 160181$, com iluminação da luz do dia (D65), e um observador de $10^{\circ}$ padronizada SCI $(8 \mathrm{~mm})$. Os parâmetros extraídos foram à luminosidade (L) e às cores primárias $\mathrm{a}^{*}$ (+ vermelha/verde -) e $\mathrm{b}^{*}$ (+ amarela/azul -). Posteriormente, o índice de amarelamento (YI) foi calculado, conforme a norma ASTM E313, por meio dos valores triestímulos X, Y e Z, utilizando a equação abaixo:

$$
\mathrm{YI}=\frac{100 *\left(C_{X} X-C_{Z} Z\right)}{Y}
$$

Os valores triestímulos $\mathrm{X}, \mathrm{Y}$ e $\mathrm{Z}$ foram calculados em função dos parâmetros a ${ }^{*}, \mathrm{~b}^{*} \mathrm{e} \mathrm{L}$, de acordo com as equações apresentadas na literatura [21]:

$$
\mathrm{a}^{*}=500 *\left[\left(\frac{X}{X_{n}}\right)^{\frac{1}{3}}-\left(\frac{Y}{Y_{n}}\right)^{\frac{1}{3}}\right] \quad \mathrm{b}^{*}=200 *\left[\left(\frac{Y}{Y_{n}}\right)^{\frac{1}{3}}-\left(\frac{Z}{Z_{n}}\right)^{\frac{1}{3}}\right] \quad \mathrm{L}^{*}=116 *\left(\frac{Y}{Y_{n}}\right)^{\frac{1}{3}}-16
$$


As constantes para uma iluminação D65 e um observador de $10^{\circ}$ são: $X_{n}=94,811 ; Y_{n}=100$; $\mathrm{Z}_{\mathrm{n}}=107,304 ; \mathrm{C}_{\mathrm{x}}=1,3013$ e $\mathrm{C}_{\mathrm{z}}=1,1498$ [21].

\section{RESULTADOS E DISCUSSÃO}

\subsection{Pó da Borracha Reciclada (SBRr)}

\section{Análise Morfológica com EDS}

Na Figura 2 (a), observa-se a micrografia obtida por MEV do pó calcinado de SBRr, enquanto a Figura 2 (b) representa o espectro de energia dispersiva (EDS) do pó de SBRr sinterizado durante $1 \mathrm{~h}$, até $600^{\circ} \mathrm{C}$.
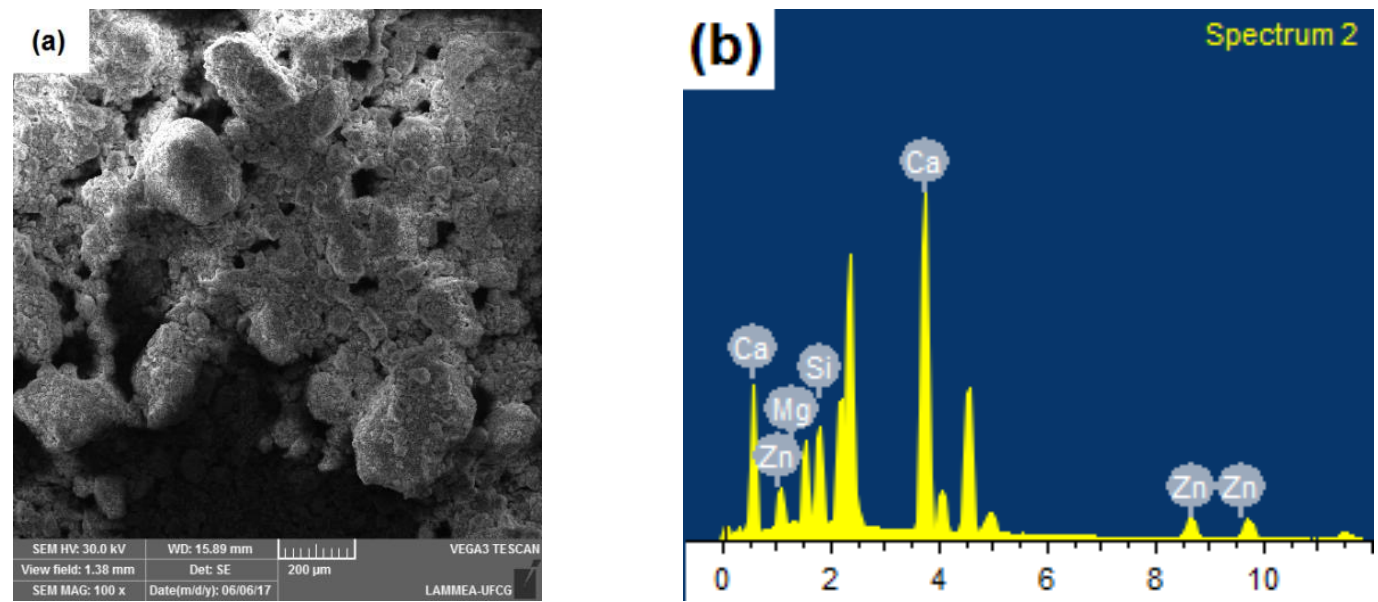

Figura 2. (a) Micrografia obtida por microscopia eletrônica de varredura (MEV) do pó calcinado da borracha reciclada (b) Espectro de energia dispersiva (EDS) do pó sinterizado da borracha reciclada de SBRr.

Verifica-se no pó sinterizado de SBRr, por análise semiquantitativa de EDS, conforme a Figura 2b, a presença de cálcio $(\mathrm{Ca})$, silício $(\mathrm{Si})$, magnésio $(\mathrm{Mg})$ e zinco $(\mathrm{Zn})$, sendo associada à presença de carbonato de cálcio $\left(\mathrm{CaCO}_{3}\right)$, sílica $(\mathrm{SiO})$, talco $\left(\mathrm{Mg}_{3} \mathrm{H}_{2} \mathrm{Si}_{4} \mathrm{O}_{12}\right)$ e óxido de zinco $(\mathrm{ZnO})$, respectivamente. Verifica-se que todas as cargas mapeadas no EDS são brancas, sendo cargas inativas o carbonato de cálcio, o óxido de zinco e o talco, enquanto que a sílica é uma carga ativa de reforço. O carbonato de cálcio e o talco, geralmente, são utilizados como cargas de enchimento em compostos de borracha. Por outro lado, o óxido de zinco é um típico ativador de vulcanização [22,23].

Termogravimetria $(T G)$

A Figura 3 mostra a curva obtida por termogravimetria (TG), para o pó do composto de borracha reciclada de SBRr. O pico em $432,6^{\circ} \mathrm{C}$, presente na curva DTG da amostra é, fisicamente, a temperatura na qual a perda de massa é máxima. 


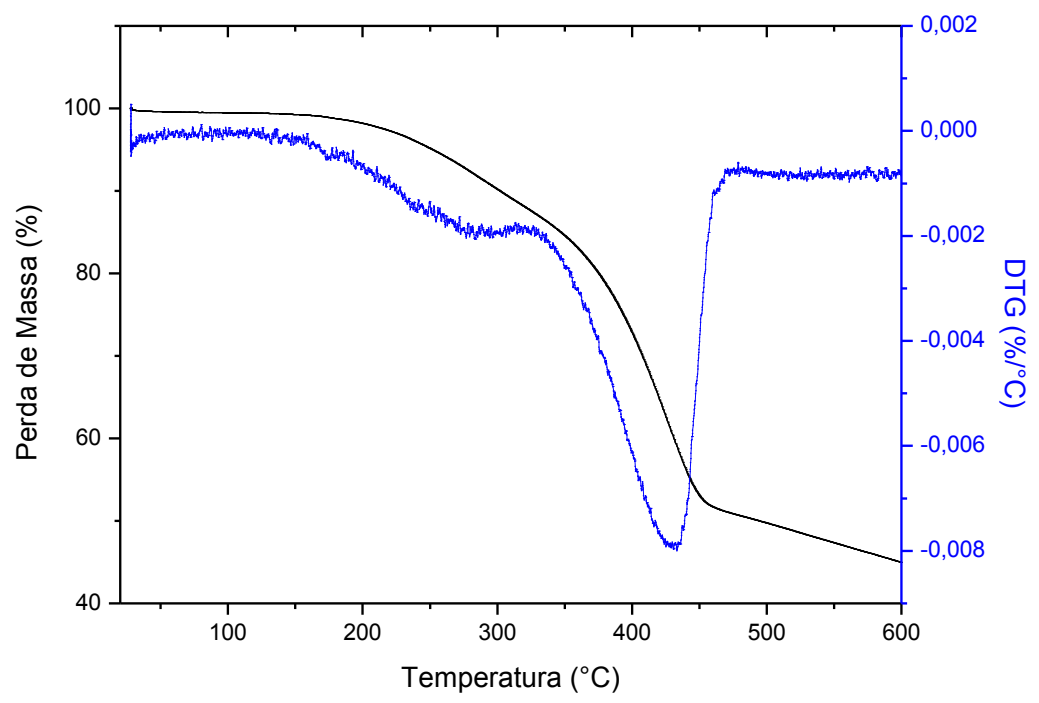

Figura 3: Curva TG/DTG do pó de borracha reciclada de $\mathrm{SBRr}$, obtida sob atmosfera de $\mathrm{N}_{2}$, a $10^{\circ} \mathrm{C} / \mathrm{min}$ e $\mathrm{m}=5 \mathrm{mg}$.

Observa-se que o composto de borracha reciclada apresentara duas etapas de decomposição térmica bem evidentes. A primeira perda de massa abaixo de $330^{\circ} \mathrm{C}$ refere-se à presença de plastificantes, antidegradantes, aceleradores e, principalmente, óleos extensores [23,34]. A segunda perda mássica, no intervalo entre 330 e $470^{\circ} \mathrm{C}$, é referente à degradação das cadeias do SBR. Observa-se, também, um teor de material residual de $44,9 \%$ ao término do processo, em $600^{\circ} \mathrm{C}$, sendo atribuído à presença das cargas de enchimento e de reforço tipicamente utilizadas em formulações de SBR da indústria de calçados, que foram confirmadas pela análise de EDS.

\subsection{Blendas Ternárias, Binárias e o HIPS}

\section{Variação da Perda de Massa}

A Tabela 2 mostra a perda de massa ao término do envelhecimento termo-oxidativo do HIPS, das blendas ternárias e binárias. Verifica-se que não há variação de massa no HIPS, indicando não ter umidade residual ou produtos voláteis. Por outro lado, de maneira geral, todas as blendas apresentaram perdas de massa mais significativas, quando comparadas ao HIPS, sendo mais pronunciada a diferença na blenda compatibilizada com SEBS. Essas perdas devem-se, provavelmente, ao fato de ocorrer uma extração dos componentes voláteis do material, devido à sensibilidade dos mesmos à temperatura. Presume-se que os aditivos plastificantes, óleos extensores e antidegradantes difundiram do interior dos corpos de prova para a superfície e, consequentemente, evaporaram ou escoaram. Os resultados de perda de massa corroboram com a primeira perda de massa observada por termogravimetria (TG).

Tabela 2: Variação de massa ao término do envelhecimento.

\begin{tabular}{lccc}
\hline \multicolumn{1}{c}{ Materiais } & Massa Inicial (0 dia) & Massa Final (60 dias) & Perda de Massa $(\Delta \mathbf{m} \%)$ \\
\hline HIPS & $4,88 \pm 0,01$ & $4,88 \pm 0,02$ & 0 \\
PS/SBRr & $5,22 \pm 0,02$ & $5,21 \pm 0,01$ & 0,19 \\
PS/SBRr/SEBS (5\%) & $5,49 \pm 0,01$ & $5,46 \pm 0,03$ & 0,55 \\
PS/SBRr/SBS (5\%) & $5,43 \pm 0,01$ & $5,42 \pm 0,01$ & 0,18 \\
\hline
\end{tabular}

\section{Resistência ao Impacto}

A Figura 4 mostra os resultados obtidos na resistência ao impacto do HIPS, da blenda PS/SBRr e das compatibilizadas com SEBS e SBS, em função dos dias de envelhecimento termo-oxidativo. 


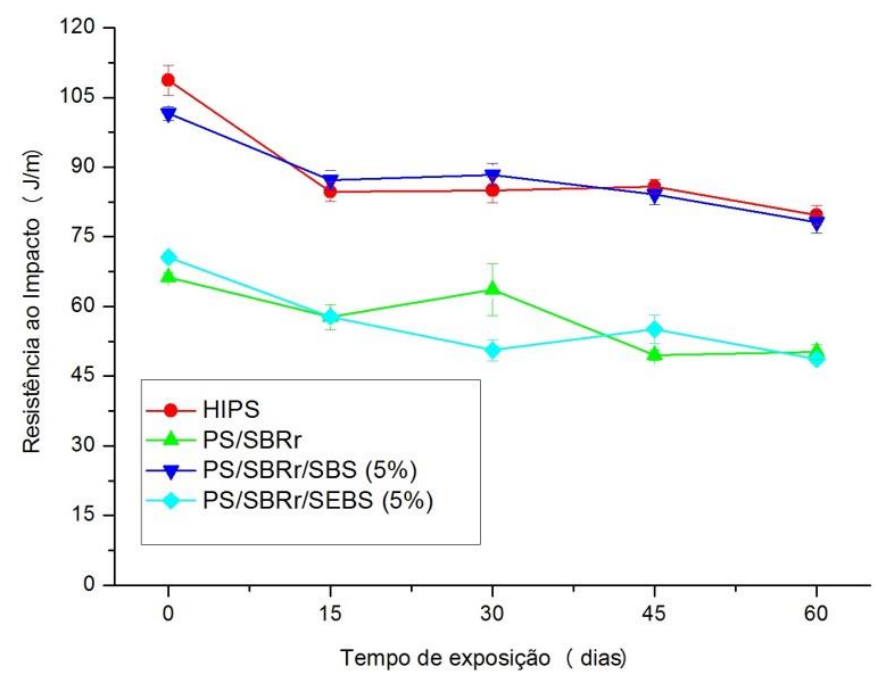

Figura 4: Resistência ao impacto do HIPS, da blenda PS/SBRr e das compatibilizadas submetidas ao envelhecimento termo-oxidativo.

Verifica-se que, dentro dos materiais não-expostos (0 dia) a termo-oxidação, o HIPS apresentou a mais alta resistência ao impacto, quando comparada às blendas, independentemente de ser compatibilizada ou não. A presença de 5\% de SEBS não se mostrou eficaz em melhorar a compatibilidade da blenda PS/SBRr, uma vez que ficou no mesmo patamar da resistência ao impacto da blenda não compatibilizada. Por outro lado, observa-se que, ao adicionar 5\% do copolímero SBS, o mesmo proporcionou um aumento considerável na resistência ao impacto, chegando a valores equiparáveis ao HIPS comercial. Presume-se, então, que, como ambos os compatibilizantes SEBS e SBS possuem grupos terminais químicos à base de estireno, o desempenho diferenciado está relacionado ao bloco elastomérico central. O SEBS apresenta um bloco central insaturado de etileno/butileno, que não é miscível com o PS ou o SBRr, enquanto que o SBS é constituído por um bloco central de butadieno, que é semelhante, quimicamente, com o bloco elastomérico do SBRr e, portanto, favorece melhores resultados de resistência ao impacto.

A Figura 4 mostra que os valores da resistência ao impacto diminuem, significativamente, nos 15 dias iniciais do envelhecimento termo-oxidativo, para todas as amostras, sendo a queda mais pronunciada para o HIPS, correspondendo a 22,1\%, quando comparado ao HIPS não envelhecido. Na literatura [3], é reportado que ocorre um período de indução, quando o consumo de oxigênio ocorre numa taxa muito baixa, seguido de uma autoaceleração, até um ponto de estabilização. É exatamente durante a autoaceleração que a degradação é mais intensa, reduzindo drasticamente a propriedade mecânica.

A partir de 15 dias de envelhecimento, a variação na resistência ao impacto ocorre em uma taxa bem menor, apresentando tendência de estabilização, ou seja, não são observados efeitos deletérios significativos nessa propriedade, independentemente da amostra. O comportamento da blenda PS/SBRr/SBS e do HIPS é bastante similar, enquanto que a única alteração e inversão de comportamento foi observada aos 30 dias de envelhecimento, quando a blenda PS/SBRr aumentou a resistência ao impacto, em comparação a 15 dias. A Tabela 3 mostra a perda total da resistência ao impacto ao término do envelhecimento, em 60 dias.

Tabela 3: Variação total da resistência ao impacto ao término do envelhecimento.

\begin{tabular}{lccc}
\hline \multicolumn{1}{c}{ Materiais } & $\begin{array}{c}\text { Resistência ao Impacto } \\
(\mathbf{J} / \mathbf{m})(\mathbf{0} \text { dia })\end{array}$ & $\begin{array}{c}\text { Resistência ao Impacto } \\
(\mathbf{J} / \mathbf{m})(\mathbf{6 0} \text { dias })\end{array}$ & $\begin{array}{c}\text { Perda da Resistência ao } \\
\text { Impacto }(\boldsymbol{\%})\end{array}$ \\
\hline HIPS & $108,7 \pm 3,2$ & $77,7 \pm 2,1$ & 28,5 \\
PS/SBRr & $66,3 \pm 1$ & $51 \pm 1,6$ & 23,1 \\
PS/SBRr/SEBS & $70,6 \pm 1$ & $49 \pm 1$ & 30,6 \\
$(\mathbf{5 \%})$ & & $79,1 \pm 2,4$ & 22,1 \\
\hline PS/SBRr/SBS (5\%) & $101,5 \pm 1,5$ & & \\
\hline
\end{tabular}


A miscibilidade dos componentes de uma blenda afeta a sua estabilidade de uma forma não previsível. Em alguns casos, a miscibilidade produz uma estabilização, e em outros uma desestabilização. A separação de fases e/ou a presença de agentes de compatibilização em blendas, parcialmente miscíveis ou imiscíveis, influencia a estabilidade do material, devido às diferentes interações que podem ocorrer entre os componentes nas interfases [2]. A Tabela 3 mostra que todos os materiais, ao término de 60 dias, apresentaram perda significativa nessa propriedade, denotando degradação no material. A blenda PS/SBRr/SEBS é mais suscetível à ação do processo de termo-oxidação, refletindo em uma maior perda na resistência ao impacto, ao término de 60 dias. Por outro lado, a blenda PS/SBRr/SBS apresentou uma tendência de retardar os efeitos degratativos da termo-oxidação sobre a resistência ao impacto, o que, de certa forma, tornou-se intrigante, uma vez que o SBS possui insaturações no bloco butadiênico, o que favorece o ataque oxidativo. Já o SEBS é derivado da hidrogenação, das ligações duplas do SBS, formando segmentos elastoméricos de etilenobutileno, sendo menos propenso ao ataque oxidativo [26]. Todavia, para a blenda de PS/SBRr/SEBS, é razoável sugerir que o bloco etileno-butileno (EB) atuou acelerando a degradação, bem como facilitou a migração de componentes voláteis (Tabela 2), quando comparado ao SBS. Essas blendas compatibilizadas foram formuladas com a mesma proporção de borracha reciclada (47,5\%), sendo o único diferencial delas os blocos de butadieno e etileno-butileno presentes no SBS e SEBS, respectivamente.

Resistência à Tração

A Tabela 4 mostra as propriedades de tração do HIPS, das blendas ternárias e binárias, com o envelhecimento termo-oxidativo.

Tabela 4: Propriedades de tração do HIPS, das blendas ternárias e binárias, com o envelhecimento termo-oxidativo.

\begin{tabular}{|c|c|c|c|c|}
\hline \multirow[b]{2}{*}{ Material } & \multirow[b]{2}{*}{ Ciclo (Dias) } & \multicolumn{3}{|c|}{ Propriedades } \\
\hline & & $\begin{array}{c}\text { Módulo de } \\
\text { Elasticidade (MPa) }\end{array}$ & $\begin{array}{c}\text { Resistência a Tração } \\
\text { (MPa) }\end{array}$ & $\begin{array}{l}\text { Alongamento na } \\
\text { Ruptura (\%) }\end{array}$ \\
\hline \multirow{5}{*}{ HIPS } & 0 dias & $(748,7 \pm 13,5)$ & $(25,8 \pm 2,0)$ & $(29,4 \pm 2,2)$ \\
\hline & 15 dias & $(686,3 \pm 10,9)$ & $(18,0 \pm 0,14)$ & $(19,7 \pm 1,5)$ \\
\hline & 30 dias & $(652,4 \pm 27,8)$ & $(17,6 \pm 0,12)$ & $(16,2 \pm 8,1)$ \\
\hline & 45 dias & $(675,0 \pm 14,5)$ & $(17,3 \pm 0,24)$ & $(16,9 \pm 1,5)$ \\
\hline & 60 dias & $(656,1 \pm 13,9)$ & $(17,7 \pm 0,24)$ & $(16,1 \pm 1,2)$ \\
\hline \multirow{5}{*}{$\mathrm{PS} / \mathrm{SBRr}$} & 0 dias & $(526,0 \pm 27,7)$ & $(15,3 \pm 1,0)$ & $(17,5 \pm 1,0)$ \\
\hline & 15 dias & $(410,8 \pm 23,1)$ & $(10,2 \pm 0,24)$ & $(10,3 \pm 1,0)$ \\
\hline & 30 dias & $(377,3 \pm 8,6)$ & $(10,0 \pm 0,29)$ & $(9,8 \pm 2,4)$ \\
\hline & 45 dias & $(391,0 \pm 5,5)$ & $(10,4 \pm 0,48)$ & $(10,0 \pm 2,4)$ \\
\hline & 60 dias & $(376,8 \pm 25,6)$ & $(10,0 \pm 0,18)$ & $(7,3 \pm 0,8)$ \\
\hline \multirow{5}{*}{$\begin{array}{c}\text { PS/SBRr/SBS } \\
\text { (5\% SBS) }\end{array}$} & 0 dias. & $(585,9 \pm 15,1)$ & $(18,0 \pm 1,0)$ & $(29,9 \pm 1,3)$ \\
\hline & 15 dias & $(354,8 \pm 16,4)$ & $(10,0 \pm 0,23)$ & $(18,8 \pm 0,7)$ \\
\hline & 30 dias & $(398,7 \pm 15,8)$ & $(9,6 \pm 0,16)$ & $(14,0 \pm 0,6)$ \\
\hline & 45 dias & $(384,6 \pm 14,7)$ & $(9,6 \pm 0,21)$ & $(14,6 \pm 0,6)$ \\
\hline & 60 dias & $(373,3 \pm 17,4)$ & $(9,7 \pm 0,09)$ & $(13,0 \pm 0,7)$ \\
\hline \multirow{5}{*}{$\begin{array}{c}\text { PS/SBRr/SEBS } \\
\text { (5\% SEBS) }\end{array}$} & 0 dias & $(547,3 \pm 21,2)$ & $(18,1 \pm 1,1)$ & $(17,3 \pm 1)$ \\
\hline & 15 dias & $(348,3 \pm 14,4)$ & $(9,8 \pm 0,09)$ & $(14,9 \pm 0,4)$ \\
\hline & 30 dias & $(372,0 \pm 9,1)$ & $(9,8 \pm 0,08)$ & $(11,9 \pm 0,3)$ \\
\hline & 45 dias & $(383,1 \pm 14,0)$ & $(9,9 \pm 0,05)$ & $(11,6 \pm 0,8)$ \\
\hline & 60 dias & $(359,1 \pm 30,3)$ & $(9,7 \pm 0,3)$ & $(11,0 \pm 1,5)$ \\
\hline
\end{tabular}


Assim como observado para a resistência ao impacto, verifica-se uma queda significativa para o módulo, a resistência e o alongamento sob tração, durante os 15 dias iniciais, de todos os materiais, seguido de certa estabilização nos valores das propriedades, ocorrendo pequenas oscilações até 60 dias. Nota-se que o módulo de elasticidade à tração das blendas sofreu uma forte redução nos 15 diais iniciais, provavelmente em decorrência de cisão molecular das cadeias. A blenda PS/SBRr/SEBS segue a mesma tendência da resistência ao impacto, apresentando comportamento equiparável ou abaixo da blenda não compatibilizada (PS/SBRr). Verifica-se que as blendas contendo SBS e SEBS apresentaram mecanismos similares no módulo de elasticidade, na faixa de 30 a 60 dias, uma vez que ambas aumentaram o módulo nessa faixa de exposição, quando comparado ao de 15 dias, fato este que não foi observado na blenda não compatibilizada. Provavelmente, na faixa de 30 a 60 dias, tenha ocorrido alguma reticulação, associada às duplas ligações $\mathrm{C}=\mathrm{C}$ presente no butadieno do SBRr, aumentando a rigidez. Já o HIPS apresenta uma maior estabilidade no módulo de elasticidade, uma vez que ele apresenta uma menor quantidade de polibutadieno em sua composição, sendo a queda mais acentuada nos 15 dias iniciais.

$\mathrm{Na}$ literatura [3], é reportado que as propriedades mecânicas dos materiais poliméricos, especialmente as verificadas sob tração, modificam-se sob processos de degradação, de acordo com dois aspectos principais: surgimento de ligações cruzadas ou cisão de cadeias.

Observa-se na Tabela 4 que os resultados obtidos para o módulo de elasticidade, resistência à tração e alongamento na ruptura são característicos de polímeros que sofreram degradação, provavelmente em decorrência de cisão de cadeias, provocando uma diminuição dessas propriedades de tração [27]. É comum atribuir ao polibutadieno a responsabilidade principal sobre a degradação no HIPS [28], já para as borrachas vulcanizadas, a reação de oxidação pode quebrar as ligações cruzadas de enxofre, tanto quanto as ligações da cadeia principal do elastômero [29-32], sendo o bloco butadieno do SBR o local mais propenso ao ataque oxidativo na cadeia principal.

O alongamento na ruptura mostrou ser uma propriedade mecânica bem sensível aos efeitos do envelhecimento, e os resultados da Tabela 4 evidenciam isso. O comportamento do alongamento na ruptura da blenda PS/SBRr apresenta um perfil de diminuição abrupta, chegando em 60 dias com uma queda de 58,3\%, quando comparado à blenda PS/SBRr não envelhecida. Para as blendas compatibilizadas, verifica-se uma tendência de melhores resultados desta propriedade ao término de 60 dias, em relação à blenda não compatibilizada, sendo mais pronunciada para a blenda contendo SBS. Para o HIPS, o comportamento é bem similar às blendas compatibilizadas, apresentando uma queda nos 15 dias iniciais, e, posteriormente, pequenas variações dessa propriedade. A literatura [21] mostra que a similaridade do alongamento na ruptura com a perda de resistência ao impacto, na primeira fase de envelhecimento, pode ser atribuída à degradação das partículas de borracha.

A resistência sob tração seguiu a mesma tendência das outras propriedades, para todas as blendas e o HIPS, apresentando uma queda acentuada nos 15 dias iniciais, que tende a se manter constante ao longo do envelhecimento. No geral, verifica-se na Tabela 4 que o envelhecimento praticamente não influenciou a resistência à tração após 15 dias, já que esta propriedade sofreu pequenas variações.

Índice de Amarelamento (YI)

A Figura 5 representa os resultados de índice de amarelamento (YI) em função do tempo de envelhecimento termo-oxidativo. 


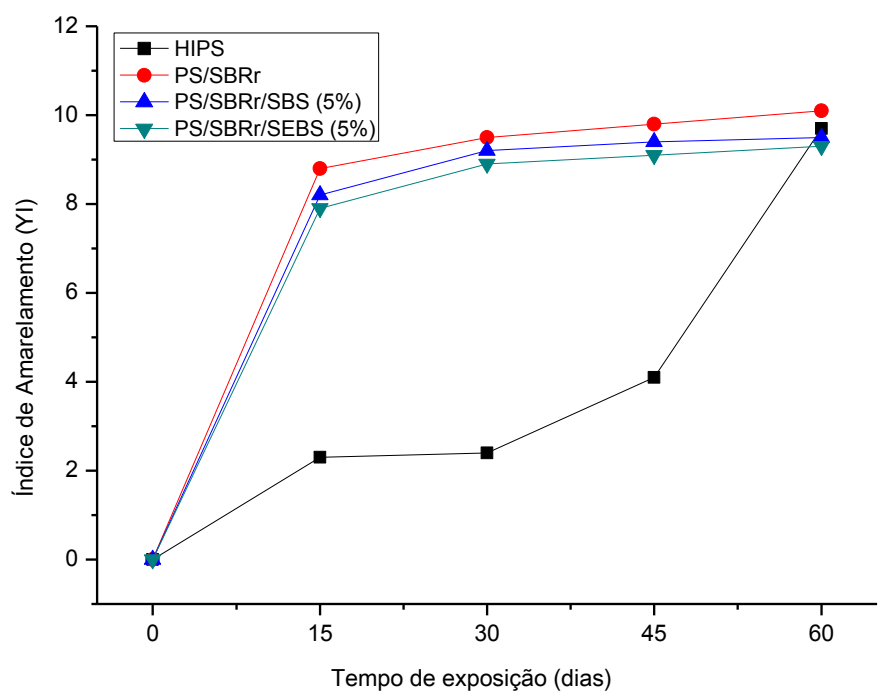

Figura 5: Evolução do índice de amarelamento (YI) do HIPS, da blenda PS/SBRr e das blendas compatibilizadas, submetidos ao envelhecimento termo-oxidativo.

Observa-se que, até 30 dias, o HIPS apresenta uma baixa taxa de amarelamento, e, posteriormente, ocorre uma intensificação no YI, chegando ao término de 60 dias com resultados equiparáveis às blendas compatibilizadas. Pode-se verificar que as blendas sofreram um aumento brusco no índice de amarelamento nos 15 dias iniciais, sendo mais pronunciado para a blenda PS/SBRr. Provavelmente, o aumento acelerado inicial do YI ocorreu devido à própria presença de aditivos que tenham migrado para a superfície, como óleos extensores, tornando a topografia amarelada ou associada a efeitos prévios de degradação, como relatado em resistência ao impacto sobre o tempo de indução. Com o decorrer do envelhecimento, a taxa de amarelamento das blendas ficou praticamente constante. Na literatura [26], as duplas ligações existentes no polibutadieno (PB) são associadas como a região propícia para ataque degradativo, favorecendo reações de oxidação no material, manifestando a coloração amarelada. A blenda PS/SBRr, por possuir a maior quantidade de borracha reciclada e, consequentemente, alto teor de insaturações no seu segmento polibutadiênico, presente no SBR, favoreceu, possivelmente, a maior taxa de amarelamento frente às compatibilizadas.

Nas blendas compatibilizadas, verificou-se que a PS/SBRr/SBS teve um índice de amarelamento levemente superior, quando comparada à blenda com SEBS, provavelmente em decorrência do SBS possuir duplas ligações em seu bloco butadiênico, enquanto o segmento elastomérico central do SEBS deriva de um bloco butadiênico que foi hidrogenado, diminuindo seu teor de insaturações. É interessante notar que, ao término de 60 dias, o HIPS ficou, praticamentre, no mesmo patamar do YI das blendas, apesar do teor de borracha ser bem menor em sua composição quando comparado com as blendas preparadas no laboratório. Presume-se que o composto de borracha reciclada apresente aditivos que podem minimizar ou estabilizar os efeitos do envelhecimento, como no caso dos antioxidantes, bem como a presença de cargas [19,35], como visto no EDS. Na literatura [33], é reportado que o índice de amarelecimento maior, em função do tempo de envelhecimento, indica, possivelmente, que a blenda sofreu uma degradação mais acentuada. Aparentemente, pelos resultados de YI, a blenda compatibilizada com SEBS teria apresentado a menor degradação entre as blendas, o que refletiria em menores perdas de propriedades. Todavia, isso não foi verificado nas propriedades mecânicas, o que reforça, possivelmente, a hipótese da migração de polímeros de baixo peso molecular para a superfície, tais como óleos extensores, apresentando tonalidade transparente a amarelada, e, consequentemente, podendo ter mascarado o resultado do índice de amarelamento.

\section{CONCLUSÕES}

Os mecanismos de degradação do poliestireno de alto impacto (HIPS) são consagrados há bastante tempo. Entretanto, a contribuição deste trabalho está na obtenção, por mistura mecânica, de blendas de poliestireno com borracha reciclada e, consequentemente, avaliação do envelhecimento termo-oxidativo dessas em relação ao HIPS. Observou-se que a borracha reciclada apresenta uma composição complexa com cargas e aditivos, indicando que esses podem minimizar perdas de propriedades, quando submetidos à tempos prolongados de exposição, mesmo utilizando altas concentrações de borracha (47,5 e 50\% em massa). De modo geral, as 
blendas apresentaram comportamento degradativo semelhante ao HIPS, uma vez que apresentam moléculas similares em suas constituições. Verificou-se que o efeito termo-oxidativo é mais deletério nas propriedades mecânicas nos 15 dias iniciais, seguido de estabilização, tanto para as blendas quanto para o HIPS. O índice de amarelamento foi mais efetivo nos primeiros 15 dias de exposição para as blendas, aspecto que pode ter resultado em maiores perdas das propriedades mecânicas. Todavia, para o HIPS, só após 30 dias os maiores valores de índice de amarelamento foram evidenciados. Observa-se a possibilidade de reutilização de uma borracha reciclada, uma vez que foram obtidos materiais com resistência ao impacto similares ao HIPS, como a blenda PS/SBRr/SBS, podendo ser considerada tenacificada na temperatura ambiente. Além disso, essa blenda apresentou uma menor perda na propriedade de impacto, comparada ao HIPS quando submetida ao envelhecimento termo-oxidativo, apesar de ser formulada com alta concentração de borracha reciclada.

\section{AGRADECIMENTOS}

Os autores agradecem à UFCG, ao CNPq pela concessão da bolsa, ao MCTI/CNPq, ao PNPD/CAPES e à São Paulo Alpargatas/PB pelo fornecimento do composto reciclado de SBRr.

\section{BIBLIOGRAFIA}

[1] CANEVAROLO JÚNIOR, S. V., Ciência dos polímeros: um texto básico para tecnólogos e engenheiros, 2 ed., São Paulo, Artliber Editora, 2006.

[2] PAOLI, M. A., Degradação e estabilização de polímeros, 2 ed., São Paulo, Chemkeys, 2008.

[3] NAVARRO, R. F., Aspectos da degradação de polímeros, 2 ed., Campina Grande, EDUFCG, 2009.

[4] SANCHEZ, E. M. S., FELISBERTI, M. A., COSTA, C. A. R., GALEMBECK, F., "Avaliação da degradação térmica e fotooxidativa do ABS para fins de reciclagem”, Polímeros: Ciência e Tecnologia, v. 13, n. 3, pp. 166-172, 2003.

[5] BORRELLY, D. F., Estudo comparativo da degradação do poliestireno e de poliestireno de alto impacto por envelhecimento natural e artificial, Dissertação de M.Sc., USP, São Paulo, SP, Brasil, 2002.

[6] COUTINHO, F. M. B., COSTA, M. P. M., GUIMARÃES, M. J. O. C., SOARES, B. G., "Estudo comparativo de diferentes tipos de polibutadieno na tenacificação de poliestireno", Polímeros: Ciência e Tecnologia, v. 17 , n. 4, pp. 318-324, 2007.

[7] ROVERE, J., CORREA, C. A., GRASSI, V. G., DAL PIZZOL, M. F., "Caracterização morfológica do poliestireno de alto impacto (HIPS)”, Polímeros: Ciência e Tecnologia, v. 18, n. 1, pp. 12-19, 2008.

[8] LUNA, C. B. B., SILVA, D. F., BASÍLIO, S. K. T., ARAÚJO, E. M., "Influência da sequência de mistura nas propriedades de blendas de PS/SBRr compatibilizada com SBS", Tecnol. Metal. Mater. Miner., v. 12, n. 1, pp.3-11, 2015.

[9] GRASSI, V. G., FORTE, M. M. C., “Aspectos morfológicos e relação estrutura propriedades de poliestireno de alto impacto", Polímeros: Ciência e Tecnologia, v. 11, n. 3, pp. 158-168, 2001.

[10] VILAPLANA, F., GREUS, A. R., KARLSSON, S., "Degradation of recycled high-impact polystyrene. Simulation by reprocessing and thermo-oxidation", Polymer Degradation and Stability, v. 91, n. 9, pp. 21632170, 2006.

[11] SARON, C., FELISBERTI, M. I., "Dynamic mechanical spectroscopy applied to study the thermal and photodegradation of poly(2,6-dimethyl-1,4-phenylene oxide)/high impact polystyrene blends", Materials Science and Engineering: A, v. 370, n. 1-2, pp. 293-301, 2004.

[12] RIBEIRO, V. F., JÚNIOR, N. S. D., RIEGEL, I. C., "Estudo da Recuperação das Propriedades de Poliestireno de Alto Impacto (HIPS) através da Incorporação de Borracha Termoplástica Tipo EstirenoButadieno-Estireno (SBS)", Polímeros: Ciência e Tecnologia, v. 22, n. 2, pp. 186-192, 2012.

[13] VILAPLANA, F., KARLSSON, S., GREUS, A. R., "Changes in the microstructure and morphology of high-impact polystyrene subjected to multiple processing and thermo-oxidative degradation", European Polymer Journal, v. 43, n. 10, pp. 4371-4381, 2007.

[14] SALMAN, S. R., AL-JARRAH, M. M. F., "Effect of Thermal Aging on the Thermal Stability of Recycled High-Impact Polystyrene", Polymer Plastics Technology and Engineering, v. 28, n. 9, pp. 1003-1007, 1989.

[15] ARAÚJO, E. M., CARVALHO, L. H., FOOK, M. V. L., D’ALMEIDA, J. R. M., "Propriedades mecânicas de blendas de PS/resíduo de borracha - Influência da concentração, granulometria e método de moldagem”, Polímeros: Ciência e Tecnologia, v. 7, n. 3, pp. 45-52, 1997. 
[16] GARCIA, P. S., SOUSA, F. D. B., CRUZ, S. A., SCURACCHIO, C. H., "Desvulcanização de borracha de pneus através de micro-ondas: efeito nas propriedades químicas termo-mecânicas", In: $13^{\circ}$ Congresso Brasileiro de Polimeros, pp. 1-4, Natal, 2015.

[17] LUNA, C. B. B., SIQUEIRA, D. D., ARAÚJO, E. M., MORAIS, D. D. S., BEZERRA, E. B., "Toughening of polystyrene using styrene-butadiene rubber (SBRr) waste from the shoe industry", Rem: Int. Eng. J., v. 71, n. 2, pp. 253-260, 2018.

[18] LUNA, C. B. B., SILVA, D. F., ARAÚJO, E. M., et al., "Blends of Polystyrene/Shoes Waste (SBRr): Influence of Mixture Sequence and Compatibilizer", Macromol. Symp., v. 383, n. 1, pp. 1800046, 2019.

[19] ESCÓCIO, V. A., MARTINS, A. F., VISCONTE, L. L. Y., NUNES, R. C. R., "Efeito do envelhecimento nas propriedades mecânicas e dinâmico-mecânicas de composições de borracha natural com mica", Polímeros: Ciência e Tecnologia, v. 14, n. 1, pp. 13-16, 2004.

[20] LUCAS, P., BABA, M., LACOSTE, J., GARDETTE, J. L., "Crosslinking of dienic elastomers resulting from ageing: X-ray diffraction and refractometry measurements", Polymer Degradation and Stability, v.76, n.3, pp.449-453, 2002.

[21] HIRAYAMA, D., Reciclagem do copolímero acrilonitrila-butadieno-estireno e do poliestireno de alto impacto oriundos de rejeitos de equipamentos elétricos e eletrônicos na forma de blendas poliméricas, Tese de D.Sc., USP, Lorena, SP, Brasil, 2015.

[22] BAETA, D.A., ZATTERA, J.A., OLIVEIRA, M.G., et al., "The use of styrene-butadiene rubber waste as a potential filler in nitrile rubber: order of addition and size of waste particles", Brazilian Journal of Chemical Engineering, v.26, n.1, pp.23-31, 2009.

[23] MASSAROTTO, M, CRESPO, J.S., ZATTERA, A. J., et al., "Characterization of Ground SBR Scraps from Shoe Industry", Materials Research, v.11, n.1, pp.81-84, 2008.

[24] PISTOR, V., ORNAGHI, F. G., FIORIO, R., et al., "Desvulcanização do resíduo de terpolímero de etileno-propileno-dieno (EPDM-r) por micro-ondas", Polímeros: Ciência e Tecnologia, v. 20, n. 3, pp. 165-169, 2010.

[25] LUNA, C. B. B., SILVA, D. F., ARAÚJO, E. M., "Efeito dos agentes de compatibilização SBS e SEBSMA no desempenho de misturas de poliestireno/resíduo de borracha de SBR", Matéria (Rio J.), v. 21, n. 3, pp. 632-646, 2016.

[26] LIBIO, I. L., GRASSI, V. G., DAL PIZZOL, M. F., et al., "Toughened polystyrene with improved photoresistance: Effects of the compatibilizers", Journal of Applied Polymer Science, v. 126, n. 1, p. 179-185, 2012.

[27] SANCHEZ, E. M. S., FERREIRA, M. M. C., FELISBERTI, M. A., "Avaliação da degradação térmica e fotooxidativa do ABS automotivo", Polímeros: Ciência e Tecnologia, v. 9, n. 4, pp. 116-122, 1999.

[28] SARON, C., Estudo da degradação térmica e fotoquímica das blendas de PPO/HIPS, Dissertação de MSc., UNICAMP, Campinas, SP, Brasil, 2001.

[29] HILLMER, K. H., SCHEELE, W., "The Degradation of Elastomers. 2. Oxidative Degradation of Natural Rubber Vulcanizates at Different Elongations and Temperatures. II", Rubber Chemistry and Technology, v. 32, n. 3, pp. $759-769,1959$.

[30] CHOI, S. S., HAN, D. H., KO, S. W., et al., "Thermal aging behaviors of elemental sulfur-free polyisoprene vulcanizates", Bull. Korean Chem. Soc., v. 26, n. 11, pp. 1853-1855, 2005.

[31] RODRIGUES, E. B., "Estudo da estabilidade das propriedades mecânicas e químicas de borracha vulcanizada com enxofre após envelhecimento térmico e oxidativo", Dissertação de MSc., USP, São Paulo, Brasil, 2010.

[32] ZANCHET, A., "Caracterização de compósitos elastoméricos com resíduos industriais de copolímero de butadieno e estireno (SBR) desvulcanizado por micro-ondas", Dissertação de MSc., USC, Caixas do Sul, Brasil, 2011.

[33] SALARI, D., RANJBAR., "Study on the recycling of ABS resins: simulation of reprocessing and thermo-oxidation", Iranian Polymer Journal, v. 17, n. 8, pp. 599-610, 2008.

[34] GARCIA, P. S., SOUSA, F. D. B., LIMA, J. A., et al., "Devulcanization of ground tire rubber: Physical and chemical changes after different microwave exposure times", Express Polymer Letters, v.9, n.11, pp.1015-1026, 2015. 
[35] LUNA, C. B. B., SILVA, D. F. , BASÍLIO, S.K.T., et al., "Desenvolvimento de blendas poliméricas visando a tenacificação dos polímeros: uma revisão", Semin., Ciênc. Exatas Tecnol., v. 36, n. 1, p. 67-80, 2015.

\section{ORCID}

Carlos Bruno Barreto Luna https://orcid.org/0000-0002-2441-7439

Felipe Belchior Calheiro Gomes https://orcid.org/0000-0001-8708-9578

Danilo Diniz Siqueira https://orcid.org/0000-0002-3533-513X

Edcleide Maria Araújo https://orcid.org/0000-0003-4906-864X 